\title{
Effect of Build Direction on Fatigue Performance of L-PBF 316L Stainless Steel
}

\author{
Chenfan Yu ${ }^{1} \cdot$ Yuan Zhong ${ }^{2} \cdot$ Peng Zhang ${ }^{3} \cdot$ Zhenjun Zhang $^{3} \cdot$ Congcong Zhao ${ }^{1}$ ' Zhefeng Zhang ${ }^{3} \cdot$ Zhijian Shen $^{1,2}$. \\ Wei Liu ${ }^{1}$
}

Received: 11 May 2019 / Revised: 10 August 2019 / Published online: 17 January 2020

(c) The Chinese Society for Metals (CSM) and Springer-Verlag GmbH Germany, part of Springer Nature 2020

\begin{abstract}
The microstructure and fatigue and tensile properties of $316 \mathrm{~L}$ stainless steel fabricated via laser powder bed fusion (L-PBF) were investigated. Two 316 stainless steel specimens with different loading directions which are either perpendicular to or parallel to building direction were prepared by L-PBF process. The results of X-ray diffraction tomography showed that there was no significant difference in morphology and size/distribution of the defects in the HB and VB samples. Since long axis of columnar grains is generally parallel to the build direction, the fatigue crack encounters more grain boundaries in VB samples under cyclic loading, which led to enhanced fatigue resistance of VB samples compared with HB sample. In contrast to HB sample, the VB sample has a higher fatigue strength due to a higher resistance to localized plastic deformation under cyclic loading. The differences in fatigue properties of L-PBF 316L SS with different build directions were predominantly controlled by solidification microstructures.
\end{abstract}

Keywords Austenitic stainless steel $\cdot$ Laser powder bed fusion $\cdot$ Tensile properties $\cdot$ Fatigue strength

\section{Introduction}

Laser powder bed fusion (L-PBF) technology can provide more possibilities for manufacturing individualized and complex geometrical components due to its high design flexibility and high-degree forming accuracy in comparison with other metal additive manufacture methods [1]. In addition, no need of extra molds or tooling and its high material utilization ratio have made L-PBF to be an ecofriendly industrial choice. Therefore, L-PBF technology has attracted much attention in various industrial applications

Available online at http://link.springer.com/journal/40195

Wei Liu

Liuw_tsinghua@163.com

1 State Key Laboratory of New Ceramic and Fine Processing, School of Materials Science and Engineering, Tsinghua University, Beijing 100084, China

2 Department of Materials and Environmental Chemistry, Arrhenius Laboratory, Stockholm University, 10691 Stockholm, Sweden

3 Laboratory of Fatigue and Fracture for Materials, Institute of Metal Research, Chinese Academy of Sciences, Shenyang 110016, China such as the biomedical, aerospace and nuclear plant fields [2-5]. Besides, L-PBF parts exhibit comparable mechanical properties under quasi-static loading in contrast to their traditionally fabricated counterparts [6-9]. However, industrial breakthrough of L-PBF parts is still plagued by defects, heterogeneous microstructures and a lack of trustworthiness and durability [10, 11]. Zhang et al. [12] investigated the relationship between the processing parameters and highcycle fatigue (HCF) performance of L-PBF 316L stainless steel (SS). They found that the porosity did not impact on the HCF properties when L-PBF 316L kept a high relative density. Niendorf et al. [13] investigated the HCF behavior of 316L SS and Ti6Al4V thoroughly and found that the fatigue damage behavior of Ti6Al4V was dominated by the processinduced pores, whereas the fatigue behavior of $316 \mathrm{~L}$ was strongly affected by its monotonic strength. Ductile 316L SS exhibited a great capacity to relieve local stress concentration caused by pore defects. The relationship between HCF property and building direction of L-PBF AlSi10Mg samples was investigated by Erhard et al. [14]. They found that the fatigue resistance in $0^{\circ}$ direction (the longitudinal axis of the samples was parallel to the $x-y$ plane) was higher than that in $45^{\circ}$ or $90^{\circ}$ directions (perpendicular to the $x-y$ plane). The parallel distributed pores or regions of non-melted 
powder (lack of fusion) primarily formed between layers were responsible for the higher fatigue resistance in $0^{\circ}$ direction. Similar results were also observed in AlSi10Mg [14] and L-PBF 17-4 PH SS [15] alloys. Un-melted regions with relatively large size and irregular shape formed between the layers were much more detrimental to the fatigue property in vertical specimens than those in horizontally built samples, because they provided higher stress concentrations under cyclic loading. Meanwhile, it is found that the solidification microstructures drastically affected the fatigue crack growth behavior. Elongated columnar grains generally oriented in build orientation led to different fatigue crack growth rates in L-PBF 316L samples manufactured with different build orientations [16].

Fatigue fracture is mostly localized structural damage process under cyclic loading, and the fatigue crack initiation is sensitive to pore defects and microstructural heterogeneity. A comprehensive understanding on the fatigue cracking mechanism, rapid solidification microstructures and shape/ size/distribution of pore defect is crucial to elevate the fatigue resistance of L-PBF materials. Build orientation of L-PBF parts may have great impact on defect directionality, grain morphology and texture and then significantly influenced eventual mechanical properties. At the same time, the

Table 1 Parameters employed in the laser powder bed fusion process

\begin{tabular}{llll}
\hline $\begin{array}{l}\text { Laser power } \\
(\mathrm{W})\end{array}$ & $\begin{array}{l}\text { Scanning speed } \\
(\mathrm{mm} / \mathrm{s})\end{array}$ & $\begin{array}{l}\text { Hatching dis- } \\
\text { tance }(\mu \mathrm{m})\end{array}$ & $\begin{array}{l}\text { Powder layer } \\
\text { thickness }(\mu \mathrm{m})\end{array}$ \\
\hline 195 & 850 & 100 & 20 \\
\hline
\end{tabular}

correlation between anisotropic tensile properties (strength, ductility) and fatigue behavior of L-PBF $316 \mathrm{~L}$ is required for evaluating the fatigue resistance in the presence of imperfections. Consequently, the objective of this study was to address the effect of build orientation on processing defects, microstructures, tensile properties and fatigue properties of L-PBF 316L SS.

\section{Materials and Experimental Procedure}

\subsection{L-PBF Process}

Raw powders for the laser powder bed fusion process were purchased from Carpenter (Carpenter Powder Products $\mathrm{AB}$, Torshälla, Sweden). The laser melting procedure was implemented using an EOSINT M270 system (EOS GmbH, Krailling, Germany). The energy density during the laser powder bed fusion process was $110 \mathrm{~J} / \mathrm{mm}^{3}$ and the laser scan direction rotated $90^{\circ}$ between sequential powder layers. Specific parameters of the L-PBF process are shown in Table 1. Blocks with a dimension of $62 \mathrm{~mm} \times 12 \mathrm{~mm} \times 10 \mathrm{~mm}$ were built horizontally (building direction perpendicular to the loading direction, HB sample) and vertically (build direction along the loading direction, VB sample), as illustrated in Fig. 1a.

\subsection{Mechanical Tests}

Dog-bone-shaped specimens for mechanical tests were machined from the as-built L-PBF specimens. The

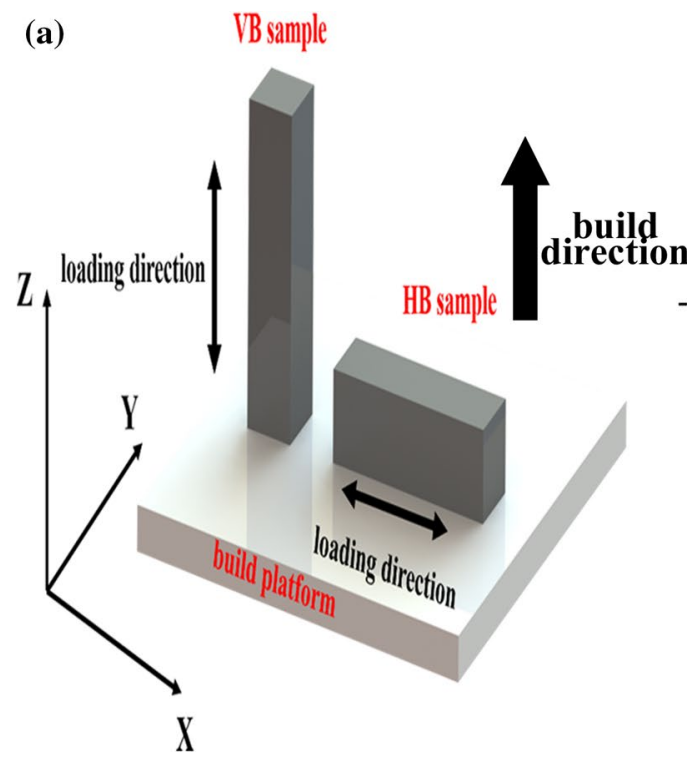

(b)

Fig. 1 a Illustration of build orientation and loading direction of HB and VB samples; the loading direction is parallel to build direction for VB sample and the loading direction is vertical to build direction for HB sample; $\mathbf{b}$ size and geometry of sample for mechanical tests 
gauge dimension of the mechanical test specimens is $10 \mathrm{~mm} \times 6 \mathrm{~mm} \times 5 \mathrm{~mm}$. As for L-PBF samples with different build orientations, three tests were taken for tensile properties. The dimension and geometry of the specimen for the mechanical tests are shown in Fig. 1b. Uniaxial tensile tests were undertaken with a nominal strain rate of $1 \times 10^{-3} \mathrm{~s}^{-1}$ under ambient condition. Stress-controlled fatigue tests with a sinusoidal wave load were performed on an Instron 8801 universal testing machine. The frequency is $30 \mathrm{~Hz}$ and the fatigue ratio is -1 . The morphology of the fracture surface, microstructures of the as-built specimen and grain orientation features were characterized using a Tescan Mira 3LMH scanning electron microscope (SEM, Tescan, Brno, Czech Republic), equipped with an electron backscatter diffraction (EBSD) detector (Oxford Instruments, Oxford, UK). An HKL Nordlys orientation imaging microscope system was used for microstructural analysis. Specimens for SEM characterization were mechanically ground, polished and then etched using $90 \%$ alcohol and $10 \%$ perchloric acid for $60 \mathrm{~s}$.

\subsection{X-ray Diffraction Topography}

The distribution and morphology of the defects were detected using a Versa XRM-500 three-dimensional X-ray diffraction tomography (3D-XRT) system with an area resolution of $1.07 \mathrm{~mm}$ per pixel, and IPWIN 6 software was used to automatically characterize the pore defects. The sampling direction of the cylindrical specimens used for XRT characterization was perpendicular to the loading direction for each sample. The size of the cylindrical sample for XRT is $\phi 2 \mathrm{~mm} \times 2 \mathrm{~mm}$.

\section{Results}

\subsection{Heterogeneous Microstructures}

The microstructures of L-PBF 316L stainless steel characterized by scanning electron microscope (SEM) are shown in Fig. 2. As shown in Fig. 2a, the half-elliptic melt pools can be clearly observed on the longitudinal section of the as-built specimen. The staggered melt traces (indicated by black dotted lines) can be seen from top side according to Fig. $2 b$. The distance between adjacent melt tracks is about $90 \mu \mathrm{m}$, which is approximately equal to the hatching distance. The size and shape of the melt pool are not uniform due to the instability of melt pool, as shown in Fig. 2a. The morphology of the melt pools is shown in Fig. 2c, the depth of the melt pool is $40 \mu \mathrm{m}$, and the width of the melt pool is $80 \mu \mathrm{m}$. The depth of most melt pool is much larger than powder layer thickness, which indicates enough laser penetration depth and sufficient fusion of metal powders. No clear-cut defects or lackof-fusion areas are observed from SEM image. Nano-sized cellular substructures with about $500 \mathrm{~nm}$ could be observed as shown in Fig. 2d. Both hexagon and strip substructures were observed in different melt pools. And also, the orientation of strip substructures is different at different regions. The length of strip generally reaches the micron scale. The growth orientation of the cells depends on preferred crystal growth direction $(<100>$ crystallographic orientation in face-centered cubic alloy) and maximum heat flux direction [17], and the orientation of the cells grew either in the same direction or rotated $90^{\circ}$ due to epitaxial growth across the melt pool boundary. The solidification microstructure is primarily contingent on temperature gradient $(G)$ and growth rate $(R)$. The value of $G \times R$ is the cooling rate, while $G / R$ ratio controls the features of solidification microstructures. In L-PBF process, the temperature gradient could reach up to $10^{6} \mathrm{~K} / \mathrm{m}$ [9]. The value of $G / R$ is above equiaxed grain solidification and planar solidification is suppressed, which is consistent with the results as shown in Fig. 2 d.

The EBSD orientation results are obtained from top side ( $X Y$ plane in Fig. 1a) and longitudinal section ( $X Z$ plane in Fig. 1a) of both HB and VB samples. For HB sample, grains lie up neatly along fusion line on the top side as shown in Fig. 3b. However, the morphology of grains on the top side of VB sample varies probably due to the uneven cyclic thermal history. As shown in Fig. 3c, d, the most prominent microstructural feature on $X Z$ plane is the elongated columnar grains along build direction. The columnar grain could be hundreds of micrometers in length. During the L-PBF process, the laser beam traverses the top of the deposited metal powder, and a micrometer-scale molten pool is formed. Loose metal powder particles around this molten pool tremendously inhibit the heat transfer, and a partial melt-back of the previously formed underlying layers occurs [18]. Furthermore, localized heat input causes significant directional cooling and solidification. Thus, the nucleation of a solid grain from a liquid in contact with a metal matrix or previously solidified material during the L-PBF process is generally referred to as epitaxial solidification and results in elongated columnar grains as shown in Fig. 3. The textures of both HB and VB samples are not evident as shown in Fig. 3, probably due to the complicated thermal history caused by layer-by-layer rotated laser scanning strategy.

\subsection{Tensile and Fatigue Properties}

Figure $4 \mathrm{a}$ shows the tensile engineering strain-stress curves of the HB and VB samples. The ultimate tensile strengths of the HB and VB sample are $677.4 \pm 13.44$ and $576.3 \pm 2.97 \mathrm{MPa}$, respectively. The elongations to failure of the HB and VB sample are $53.15 \pm 5.73 \%$ and $71.9 \pm 0.57 \%$, respectively. Moreover, the static toughness, which represents the energy of mechanical deformation per unit volume prior to fracture and reflects the resistance to 

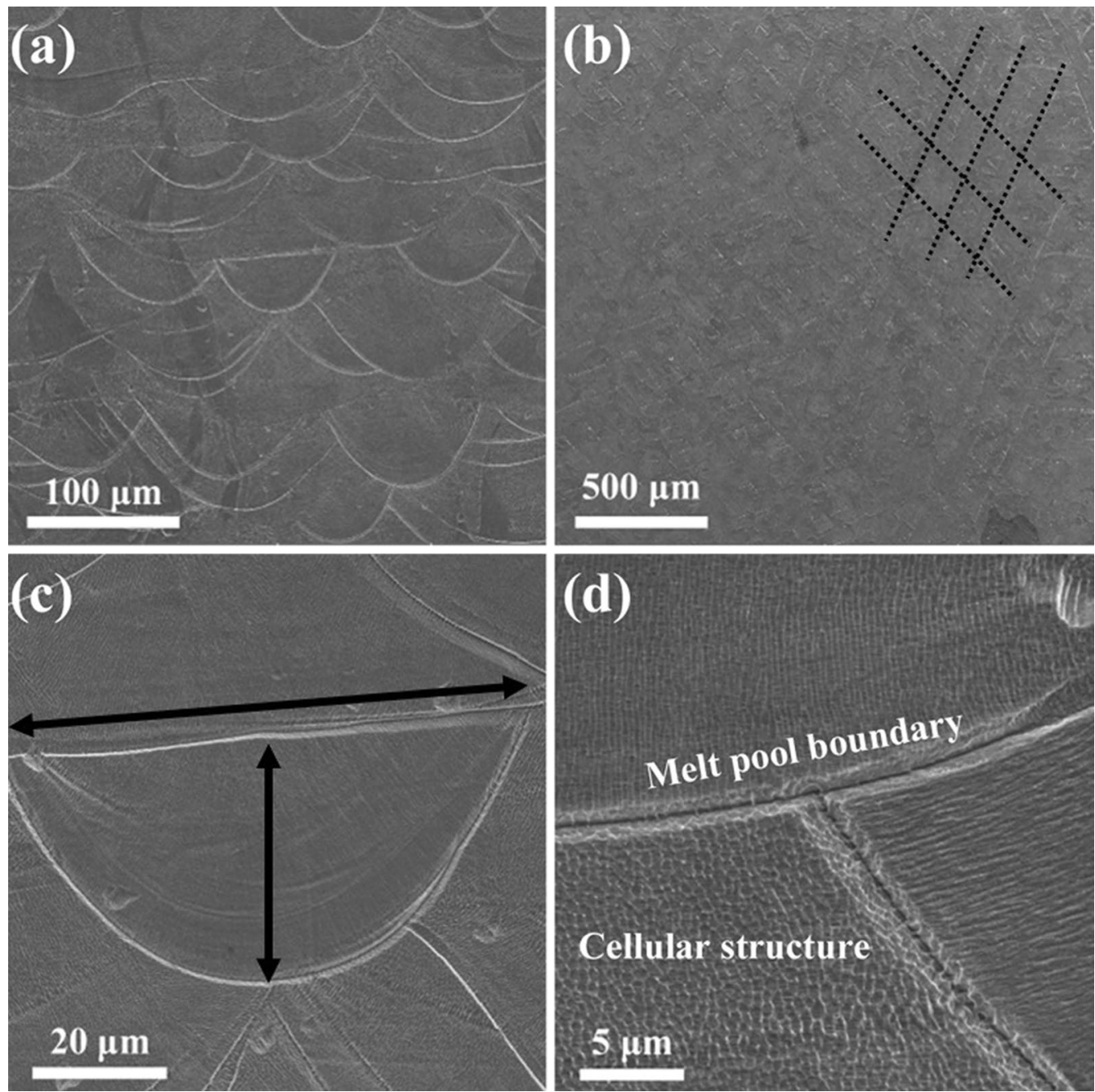

Fig. 2 Microstructures of L-PBF 316L stainless steel built vertically: a lateral side ( $X Z$ plane); b top side ( $X Y$ plane); $\mathbf{c}$ melt pool morphology; d cellular structures with different orientations near the melt pool boundary

deformation and cracking, is also considered for both samples. By estimating the integral area underneath the engineering stress-strain curves in Fig. 4a, the results show that the average static toughnesses of $\mathrm{HB}$ and VB samples are $337.8 \times 10^{6} \mathrm{~J} / \mathrm{m}^{3}$ and $431.2 \times 10^{6} \mathrm{~J} / \mathrm{m}^{3}$, respectively. It can be seen that HB sample exhibits enhanced tensile strength, while the VB sample exhibits increased ductility and toughness, demonstrating a significant effect of build orientation on tensile properties of L-PBF 316L stainless steel. Therefore, L-PBF 316L stainless steel shows an apparent anisotropic tensile property, which is mainly caused by the different rapid solidification microstructures along different building orientations. The anisotropic tensile property of L-PBF 316L SS was also reported in other studies [19-21]. Fracture surfaces of L-PBF 316L stainless steel after the tensile test are provided in Fig. 5. The difference in fracture morphology could be distinguished from the tensile fracture surface of HB and VB samples. The fracture surface of the
HB sample is smoother than that of the VB sample, which indicates better ductility of the VB sample. Obviously, tearing rupture and dimple fracture morphologies are found on the tensile fracture surface of both HB and VB samples as shown in Fig. 5c, d. The tensile fracture mode of L-PBF $316 \mathrm{~L}$ stainless steel is a manner of ductile and brittle mixed fracture.

The cycles of fatigue failure plotted against the total stress amplitudes of the HB and VB samples, i.e., the $S-N$ curves, are shown in Fig. 4b. The fatigue strengths of the $\mathrm{HB}$ and VB specimens are 190 and $210 \mathrm{MPa}$, respectively. The stress-controlled fatigue behavior could be empirically described by Basquin's equation:

$\sigma_{\mathrm{a}}=\sigma_{\mathrm{f}}^{\prime}(2 N)^{\mathrm{b}}$,

where $\sigma_{\mathrm{a}}$ is the cyclic stress amplitude, $\sigma_{\mathrm{f}}^{\prime}$ is the fatigue strength coefficient, $N$ is the number of cycles to failure and 

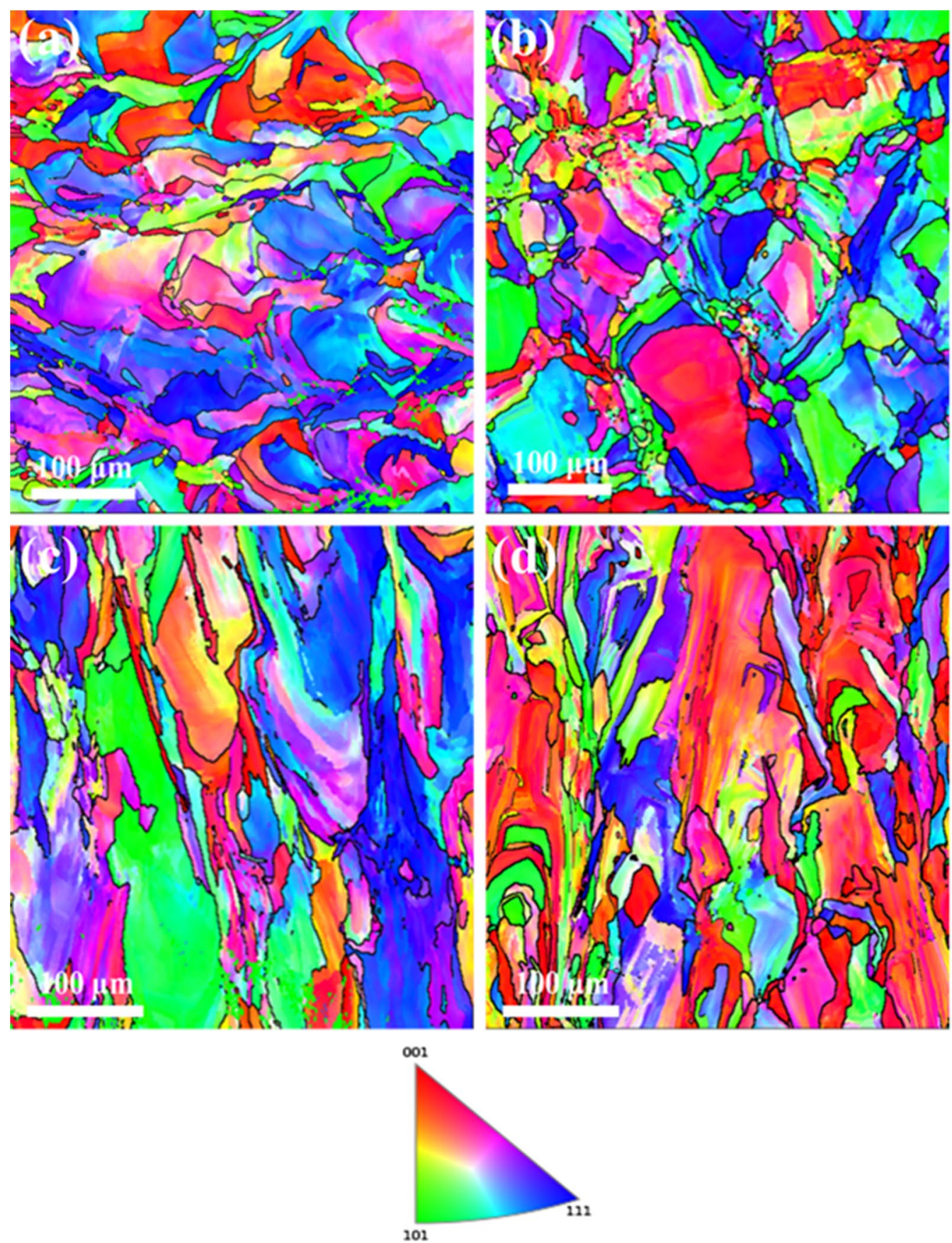

Fig. 3 Electron backscatter diffraction (EBSD) inverse-pole figure (IPF) map of each side of as-built L-PBF 316L SS sample fabricated via different build orientations: a top side of VB sample; $\mathbf{b}$ top side of HB sample; $\mathbf{c}$ lateral side of VB sample; $\mathbf{d}$ lateral side of HB sample. Bottom: IPF legend

$b$ is the fatigue strength exponent (or Basquin exponent). According to Eq. (1), $\sigma_{\mathrm{f}}^{\prime}$ and $b$ are related to the intercept on the ordinate and the slope of a logarithmic $S-N$ curve when $N$ is equal to 0.5 . Thus, the fatigue strength exponent $(b)$ and fatigue strength coefficient $\left(\sigma_{\mathrm{f}}^{\prime}\right)$ could be obtained from the $S-N$ curve, as listed in Table 2. In general, there is an intimate correlation between $\sigma_{\mathrm{f}}^{\prime}$ and the tensile strength, $\sigma_{\mathrm{f}}^{\prime}$, is roughly equivalent to the ultimate tensile strength $\left(\sigma_{\mathrm{UTS}}\right)$ for steel materials [22]. It can be seen that the fatigue strength coefficient $\left(\sigma_{\mathrm{f}}^{\prime}\right)$ of L-PBF 316L SS was not equal to the UTS. However, the fatigue strength coefficient of HB sample was higher than that of VB sample, which was consistent with the result of UTS. Therefore, the Basquin's equation could be used to describe the fatigue behavior of L-PBF 316L SS. 

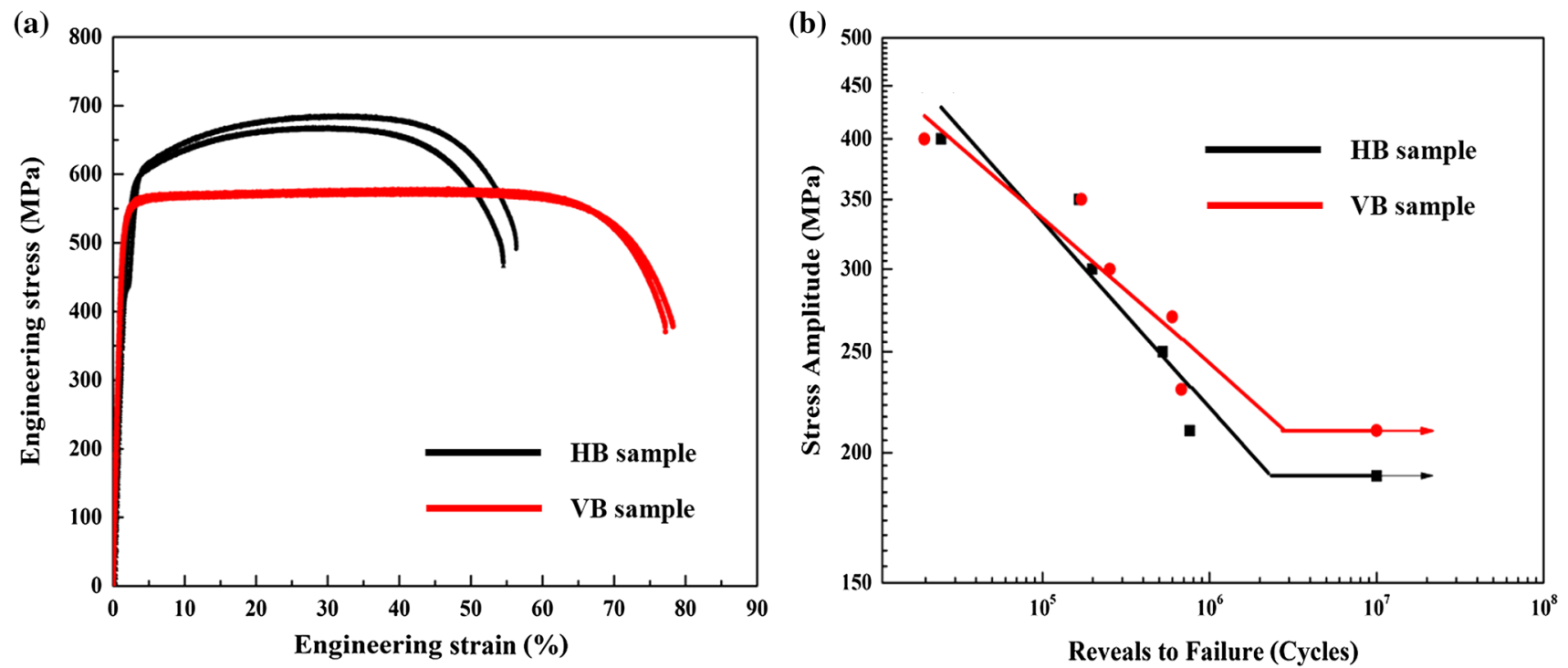

Fig. 4 a Tensile engineering strain-stress curves of 316L SS fabricated by L-PBF; b $S-N$ curves showing the relationship between fatigue life $\left(N_{\mathrm{f}}\right)$ and total stress amplitude $(\Delta \sigma / 2)$ of 316L SS specimens fabricated with different build orientations

The fatigue fracture surfaces are shown in Fig. 6, and the region of fatigue crack initiation, fatigue crack propagation and instant rupture can be distinguished on the fractograph of fatigued HB sample at stress amplitude of $400 \mathrm{MPa}$ and fatigued VB sample at stress amplitude of $300 \mathrm{MPa}$, as shown in Fig. 6a, b. Fatigue striation is typical morphology on the fatigue fracture surface. The fatigue striation morphologies are shown in Fig. 6c, d.

\subsection{Defects Characterization}

The morphology and size/distribution of pore defects in $\mathrm{HB}$ and VB specimens detected by XRT are shown in Fig. 7. As shown in Fig. 7a, b, the distribution of the defects in both HB and VB samples is dispersive and uniform. The spherical and ellipsoidal defects are the most prevalent, as shown in Fig. 7c, d. No large size and irregular voids, which were more disadvantageous to fatigue performance than other types of defects, were observed in both HB and VB specimens. And also, un-melted regions are not observed in this study due to appropriate laser penetration depth and sufficient fusion. Thus, there is no significant difference in the directionalities of the defects between the HB and VB samples. The statistical results of defect size distribution are shown in Fig. 7e. The equivalent diameter of most (nearly $90 \%$ ) of the defects in the HB and VB samples is less than $10 \mu \mathrm{m}$. It can be seen from the results of XRT that the parameters used in this study are appropriate for building near-dense 316L stainless steel.

Laser powder bed fusion process is a complex physical process involving laser interaction with metal powder, metal powder movement and dynamical melt motion, and there are miscellaneous causes of the pore defects in the laser powder bed fusion process [23-25]. In terms of energy effect, insufficient energy input results in a poor fusion between the layers and un-melted regions formed. Gas pores and keyhole pores could form owing to the excessive volumetric energy density. Pore defects create stress concentration, which might trigger premature failure. The irregularly shaped pores are more detrimental to the fatigue performance than nearly spherical pores because they might provide more stress concentration under cyclic loading.

\section{Discussion}

\subsection{Effect of Microstructures on Fatigue Properties}

The total fatigue life $\left(N_{\mathrm{T}}\right)$ is conventionally assumed to be the sum of the fatigue crack initiation life $\left(N_{\mathrm{i}}\right)$ and fatigue crack propagation life $\left(N_{\mathrm{p}}\right)$ [26]. For the fatigue crack propagation process, high-angle grain boundaries could play a role hindering cracks propagation [27, 28]. Figure 8 shows the fatigue crack propagation region on the fatigue fracture surface of HB and VB specimens. The fatigue striation essentially generates during each loading cycle, and the propagation direction indicated by arrow of fatigue crack was generally perpendicular to fatigue striation. As shown in Fig. 8a, the fatigue crack propagation direction has not changed; however, as shown in Fig. 8b, the changes in fatigue crack propagation direction might be due to the fatigue crack encountering the grain boundaries.

As mentioned before, from the longitudinal side view (Fig. 3c, d), elongated columnar grains mostly grow along 

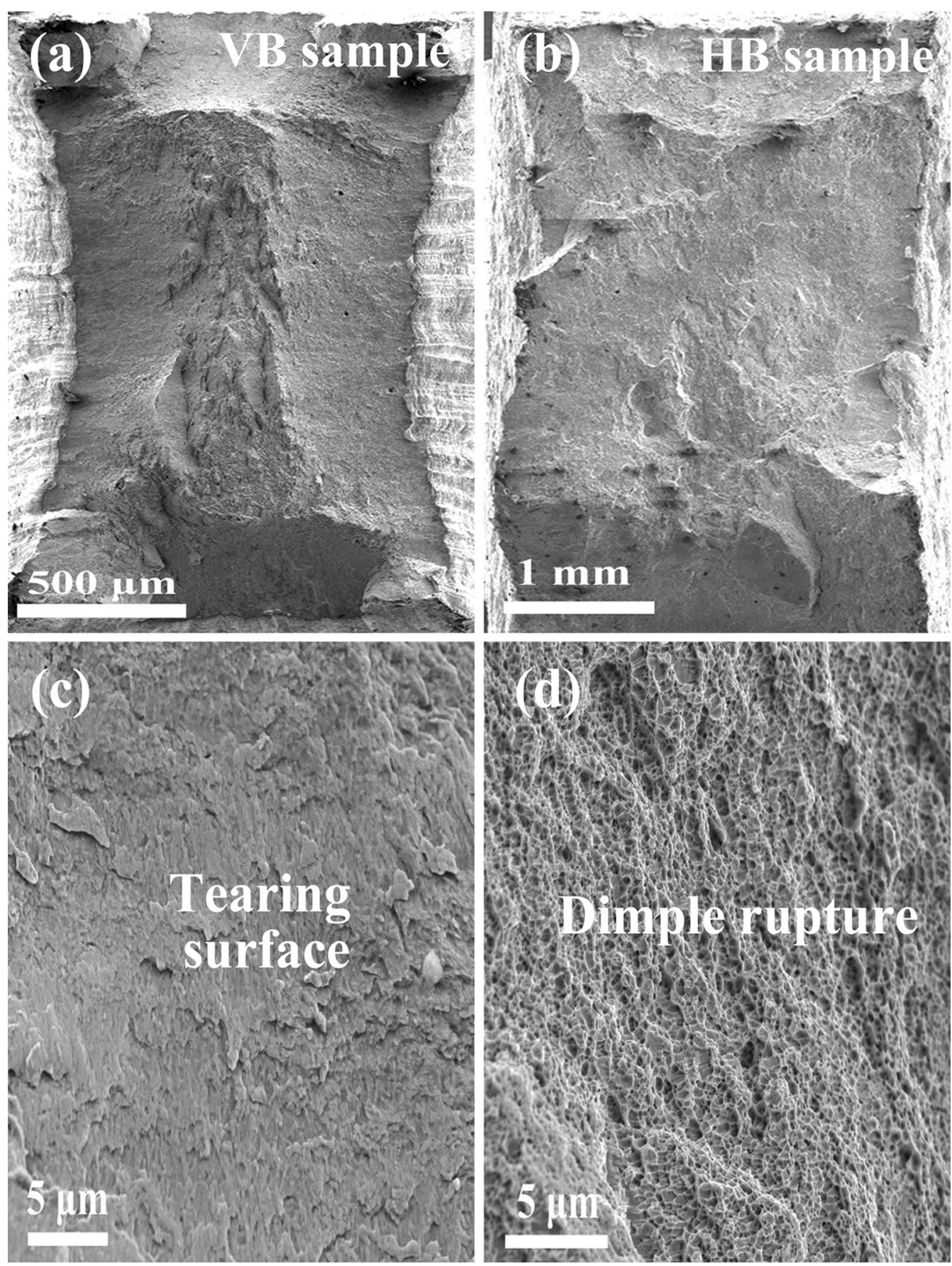

Fig. 5 Tensile fracture surfaces of L-PBF 316L SS: a VB sample; b HB sample; $\mathbf{c}$ tearing surface; d dimple rupture morphology

Table 2 Fatigue properties of L-PBF 316L SS

\begin{tabular}{llll}
\hline & $\begin{array}{l}\text { Fatigue strength } \\
\left(\sigma_{\mathrm{f}}\right)(\mathrm{MPa})\end{array}$ & $\begin{array}{l}\text { Fatigue strength coef- } \\
\text { ficient }\left(\sigma_{\mathrm{f}}^{\prime}\right)(\mathrm{MPa})\end{array}$ & $\begin{array}{l}\text { Basquin } \\
\text { exponent } \\
(b)\end{array}$ \\
\hline HB sample & 190 & 497 & -0.18 \\
VB sample & 210 & 455 & -0.14 \\
\hline
\end{tabular}

build direction due to the epitaxial growth mechanism [29, 30]. For VB sample (Fig. 9a), the loading direction is parallel to the long axis of the columnar grains and consequently fatigue crack propagation path is more tortuous caused by the resistance of grain boundaries than that of $\mathrm{HB}$ sample (Fig. 9b). The anisotropic columnar grain morphology and applied loading direction led to different fatigue crack behaviors for L-PBF 316L SS with different build orientations. 

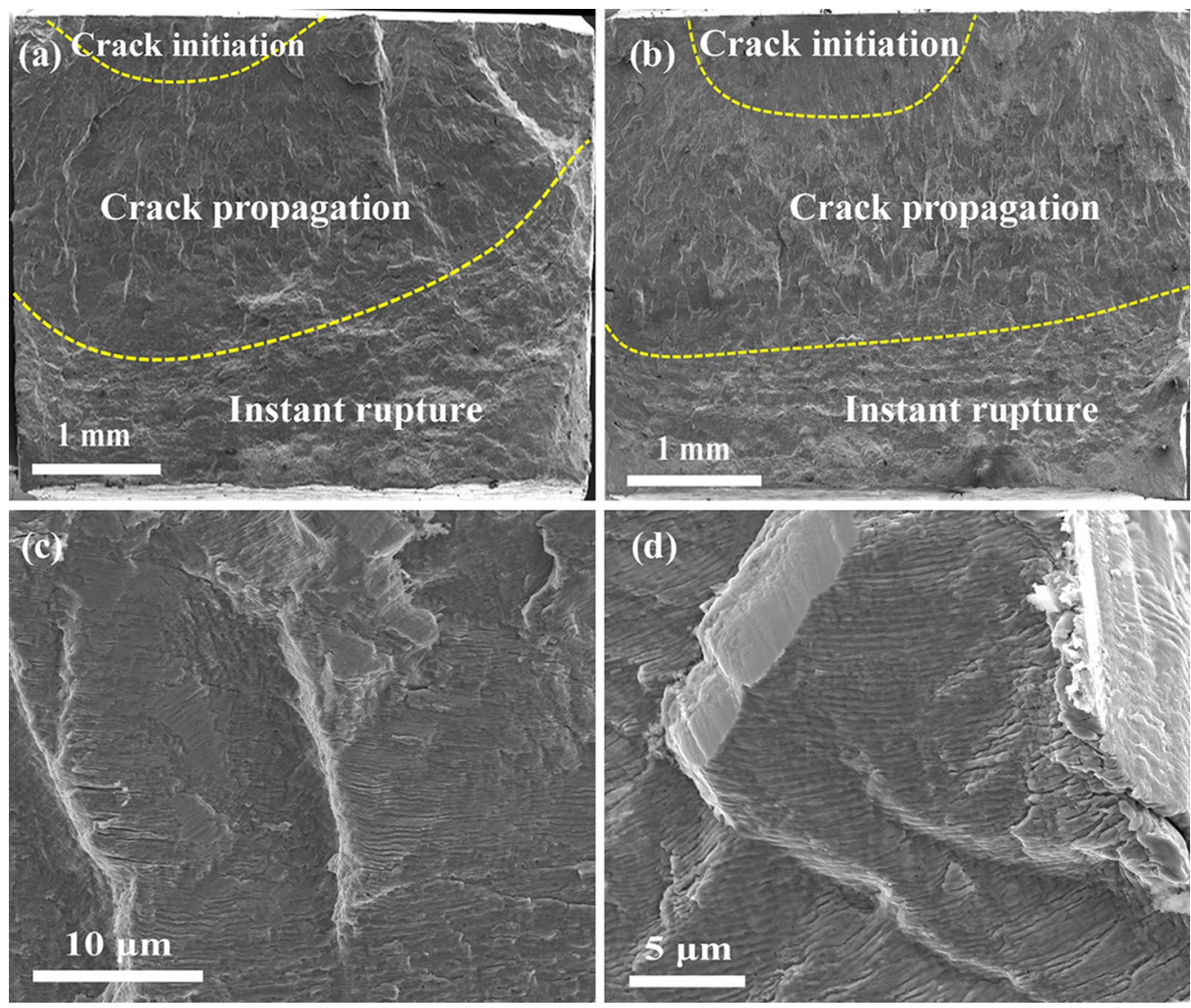

Fig. 6 Fatigue fracture surfaces of L-PBF 316L SS: a horizontally built specimen, $\Delta \sigma=400 \mathrm{MPa}, N_{\mathrm{f}}=24,713$ cycles; $\mathbf{b}$ vertically built specimen, $\Delta \sigma=300 \mathrm{MPa}, N_{\mathrm{f}}=253,003$ cycles; $\mathbf{c}, \mathbf{d}$ typical fatigue striations morphologies of $\mathbf{a}$ and $\mathbf{b}$, respectively

\subsection{Correlation Between Tensile and Fatigue Properties}

Analyzing the correlation between static mechanical properties (such as ultimate tensile strength and hardness) and fatigue strength is a simple method to evaluate the fatigue limit of materials. For ferrous metals, the linear relationship between fatigue strength $\left(\sigma_{\mathrm{w}}\right)$ and ultimate tensile strength $\left(\sigma_{\mathrm{b}}\right)$ could be described by the formula: $\sigma_{\mathrm{w}}=(0.4-0.5) \sigma_{\mathrm{b}}$ under some specific conditions [31]. However, Ohba and Murakami found that the maximum size $\left(l_{0}\right)$ of non-propagating crack decreased with the increase in static strength or hardness of steels [32]. This trend implies that with the increase in static strength or hardness, fatigue cracks of a material with high strength mostly initiate at imperfections such as defects or inclusions, rather than nucleated at persistent slip bands (PSBs) or grain boundaries (GBs) [33]. Pang et al. [34] recently established a parabolic relationship between fatigue and tensile strength in the presence of processing defects as:
$\sigma_{\mathrm{w}}=\sigma_{\mathrm{b}}\left(C-P \cdot \sigma_{\mathrm{b}}\right)$,

where $C$ is an intrinsic factor due to the effects of intrinsic defects (vacancies and dislocations) and $P$ is a fatigue sensitivity factor to processing defects (inclusions and pores). Equation (2) indicates that fatigue strength decreases with the increase in tensile strength when strength exceeds a certain value. The XRT results show that there is no difference between HB and VB samples, and thus, tensile strength should strongly affect the fatigue behavior of L-PBF 316L SS fabricated via different build orientations. As shown in Fig. 4, VB samples with a lower tensile strength and higher ductility showed enhanced fatigue strength compared with those of the HB samples. As shown in Fig. 10, fatigue cracks of L-PBF 316L SS generally initiate at the surface or the defects and fatigue damage is primarily caused by localized deformation. Increasing the work-hardening ability could effectively promote the accumulation of localized plastic deformation endurance [35], and Zhang et al. [36] defined hardened strength $\left(\Delta \sigma_{\mathrm{T}}\right)$ as the difference between maximum 

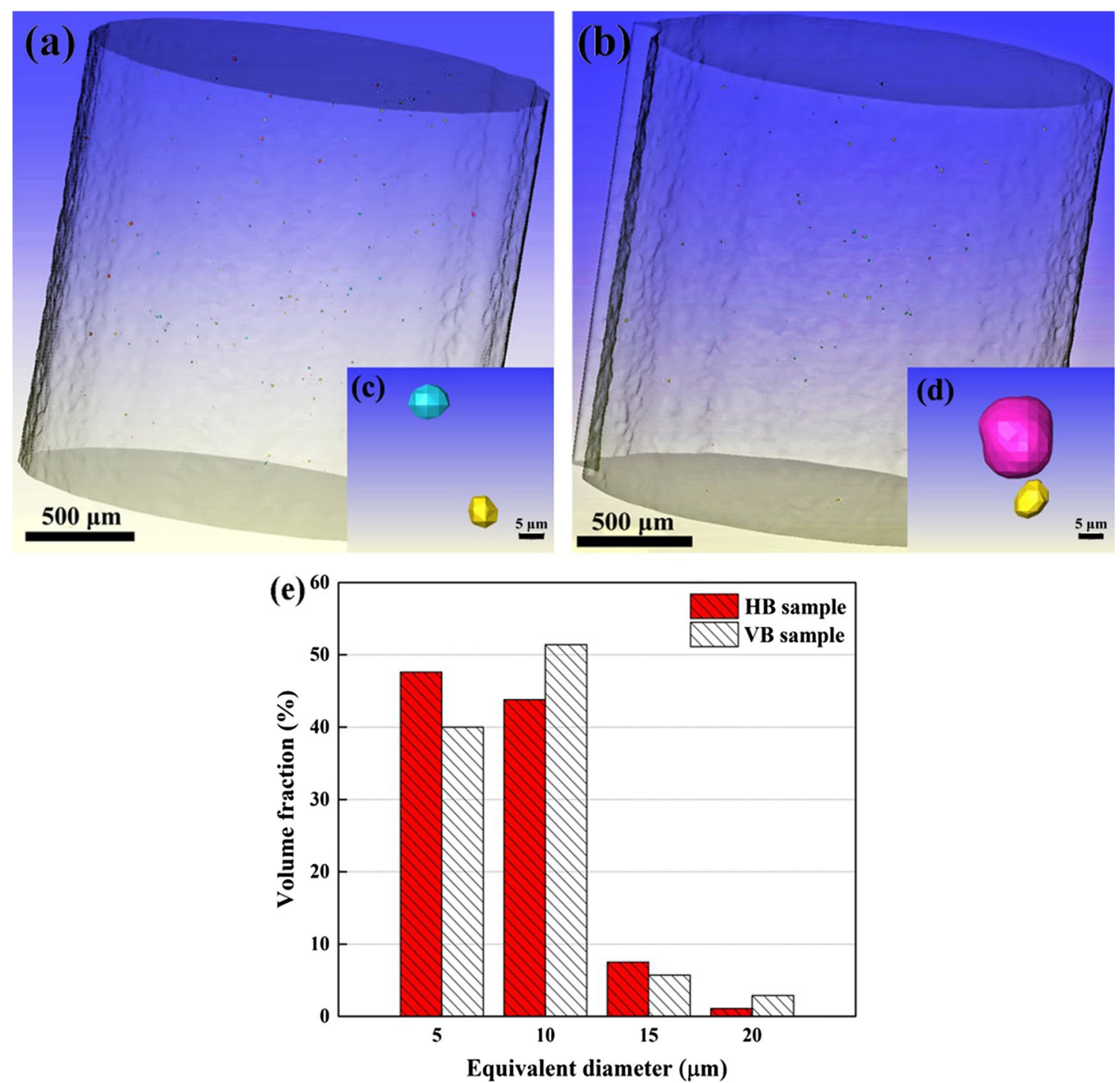

Fig. 7 Morphologies and distributions of pore defects detected by X-ray diffraction topography (XRT): a HB sample; $\mathbf{b}$ VB sample; insets $\mathbf{c}$ and $\mathbf{d}$ are magnified views of defects in $\mathbf{a}$ and $\mathbf{b}$; $\mathbf{e}$ histograms of the defect size distribution in the HB and VB samples

stress and limit of elasticity to reflect the resistance to localized plastic deformation. As shown in Fig. 11, in comparison with the HB sample, VB samples with enhanced hardened strength exhibit better deformation homogeneity and higher resistance to localized plastic deformation under cyclic loading, which led to enhanced fatigue strength.

Highly desired microstructural features could be achieved by controlling the process parameters and building orientation in the L-PBF process, which would result in various combinations of strength, ductility and toughness for L-PBF parts. The correlation between the static mechanical properties and fatigue resistance provides a method for selecting suitable mechanical properties for L-PBF 316L SS under different service environments.

\section{Conclusions}

The microstructures and tensile and fatigue properties of L-PBF 316L SS manufactured with vertical and horizontal build directions were investigated. The conclusions can be summarized as follows:

1. The fatigue behavior of L-PBF 316L SS fabricated with different build orientations is predominantly controlled by solidification microstructures.

2. For L-PBF 316L SS, elongated columnar grains generally grow along build direction, resulting in anisotropic mechanical behaviors including tensile properties and 

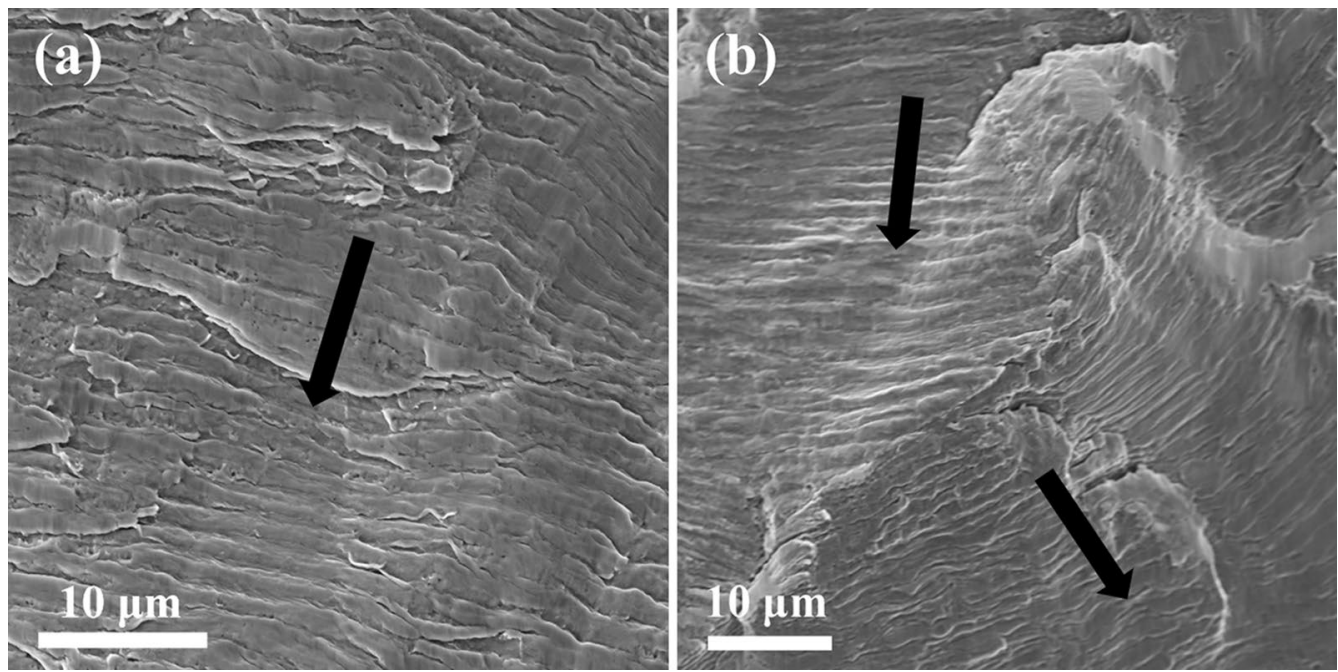

Fig. 8 Fatigue striations morphologies on fatigue propagation region: a HB sample; b VB sample. The black arrows point to the fatigue growth direction
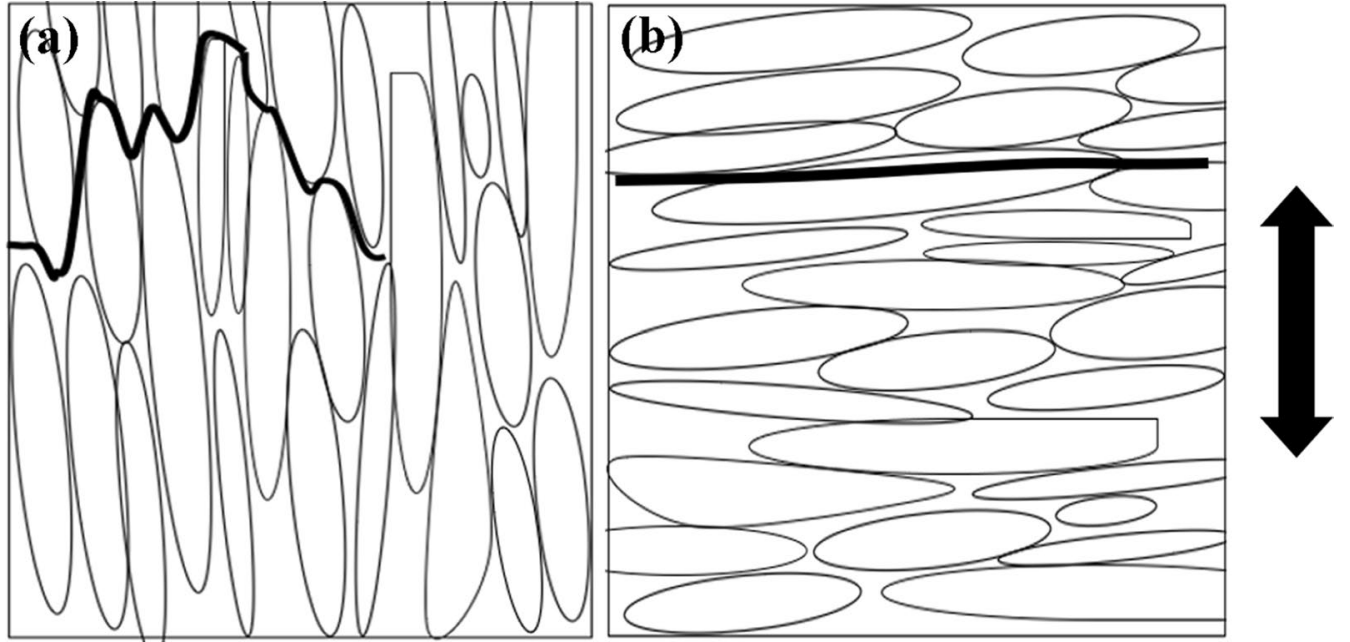

Fig. 9 Illustration of fatigue crack propagation path and typical columnar grains: a VB sample; $\mathbf{b}$ HB sample. The double-headed arrow indicates the applied loading direction

fatigue performance. When the loading direction is parallel to building direction (VB samples), fatigue crack propagation path is deflective due to the barrier of the grain boundaries, which leads to higher fatigue strength.

3. The sizes/distributions and morphologies (i.e., sphericities) of the defects detected by XRT in the HB and VB samples show no significant differences. No un-melted regions (lack-of-fusion) are observed in $\mathrm{HB}$ and VB samples and spherical and ellipsoidal defects are the most prevalent.

4. The tensile strength of HB sample is higher than that of VB sample, while VB sample exhibits better ductility and higher hardened strength $\left(\Delta \sigma_{\mathrm{T}}\right)$. VB sample exhibits a higher fatigue strength because of a better endurance to local plastic deformation than that of HB sample. 

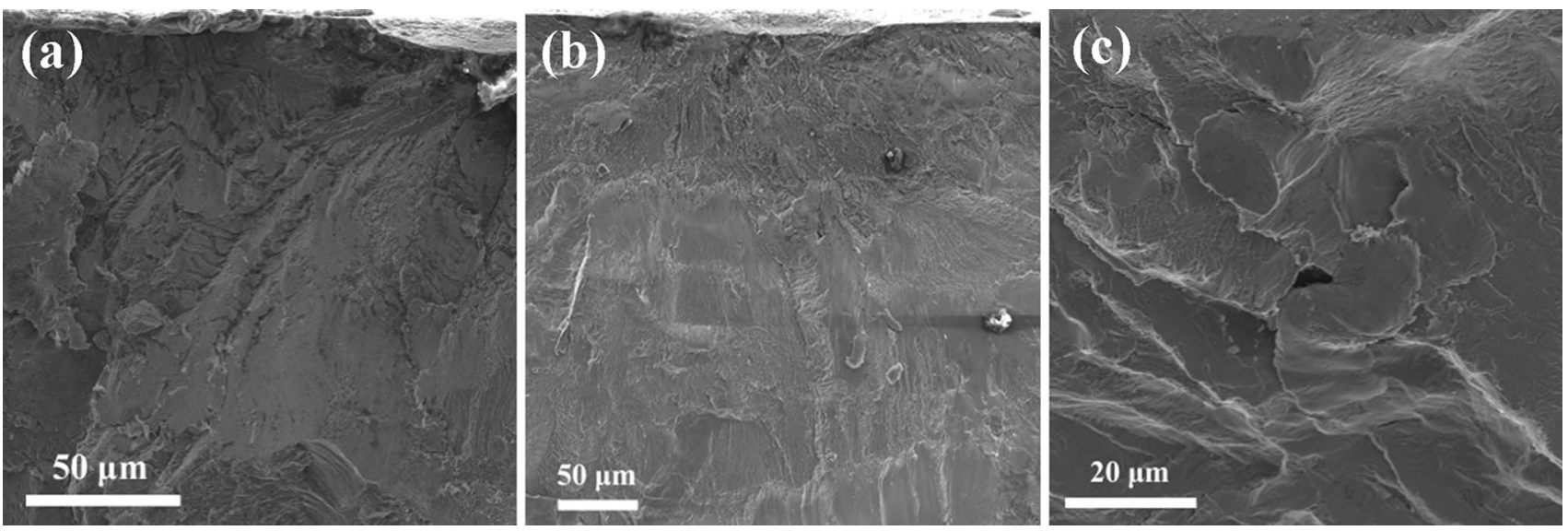

Fig. 10 Fatigue crack initiation regions: $\mathbf{a}, \mathbf{b}$ fatigue crack initiated at the surface of the tested sample; $\mathbf{c}$ fatigue crack initiated at the pore defect

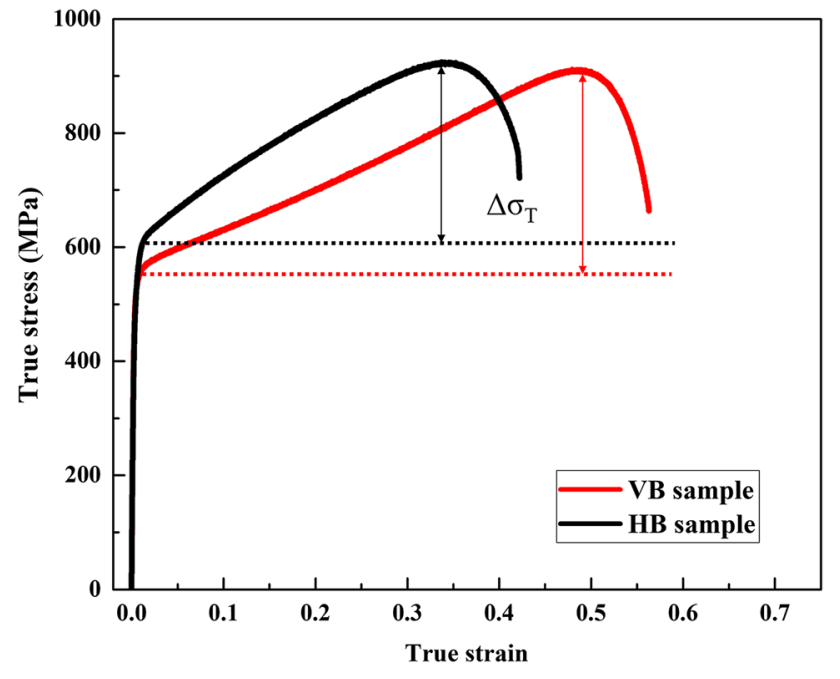

Fig. 11 True stress-true strain curves of both HB and VB samples

Acknowledgements This work was financially supported by the National Magnetic Confinement Fusion Science Program of China under Grant 2014GB117000, as well as the Joint Funds of the National Natural Science Foundation of China under Grant U1605243.

\section{References}

[1] D.L. Bourell, Annu. Rev. Mater. Res. 46(1), 1 (2016)

[2] Y.S. Hedberg, B. Qian, Z.J. Shen, S. Virtanen, I.O. Wallinder, Dent. Mater. 30(5), 525 (2014)

[3] D.D. Gu, W. Meiners, K. Wissenbach, R. Poprawe, Int. Mater. Rev. 57(3), 133 (2012)

[4] I. Yadroitsev, P. Krakhmalev, I. Yadroitsava, J. Alloys Compd. $\mathbf{5 8 3}, 404$ (2014)

[5] E.O. Olakanmi, R.F. Cochrane, K.W. Dalgarno, Prog. Mater. Sci. 74, 401 (2015)

[6] Y.M. Wang, T. Voisin, J.T. McKeown, J. Ye, N.P. Calta, Z. Li, Z. Zeng, Y. Zhang, W. Chen, T.T. Roehling, R.T. Ott, M.K.
Santala, P.J. Depond, M.J. Matthews, A.V. Hamza, T. Zhu, Nat. Mater. 17(1), 63 (2018)

[7] L. Liu, Q. Ding, Y. Zhong, J. Zou, J. Wu, Y.L. Chiu, J. Li, Z. Zhang, Q. Yu, Z. Shen, Mater. Today 21(4), 354 (2018)

[8] B. Vrancken, L. Thijs, J.-P. Kruth, J. Van Humbeeck, J. Alloys Compd. 541, 177 (2012)

[9] L. Thijs, K. Kempen, J.-P. Kruth, J. Van Humbeeck, Acta Mater. 61(5), 1809 (2013)

[10] D. Herzog, V. Seyda, E. Wycisk, C. Emmelmann, Acta Mater. 117, 371 (2016)

[11] C. Emmelmann, J. Kranz, D. Herzog, E. Wycisk, Laser additive manufacturing of metals, in Laser Technology in Biomimetics: Basics and Applications, ed. by V. Schmidt, M.R. Belegratis (Springer, Berlin, 2013), pp. 143-162

[12] M. Zhang, C.-N. Sun, X. Zhang, P.C. Goh, J. Wei, D. Hardacre, H. Li, Mater. Sci. Eng. A 703, 251 (2017)

[13] S. Leuders, T. Lieneke, S. Lammers, T. Tröster, T. Niendorf, J. Mater. Res. 29(17), 1911 (2014)

[14] E. Brandl, U. Heckenberger, V. Holzinger, D. Buchbinder, Mater. Des. 34, 159 (2012)

[15] A. Yadollahi, N. Shamsaei, S.M. Thompson, A. Elwany, L. Bian, Int. J. Fatigue 94, 218 (2017)

[16] A. Riemer, S. Leuders, M. Thöne, H.A. Richard, T. Tröster, T. Niendorf, Eng. Fract. Mech. 120, 15 (2014)

[17] Z.W. Chen, M.A.L. Phan, K. Darvish, J. Mater. Sci. 52(12), 7415 (2017)

[18] X. Zhou, K. Li, D. Zhang, X. Liu, J. Ma, W. Liu, Z. Shen, J. Alloys Compd. 631, 153 (2015)

[19] L. Hitzler, J. Hirsch, B. Heine, M. Merkel, W. Hall, A. Ochsner, Materials 10(10), 1136 (2017)

[20] W. Shifeng, L. Shuai, W. Qingsong, C. Yan, Z. Sheng, S. Yusheng, J. Mater. Process. Technol. 214(11), 2660 (2014)

[21] Y.D. Im, K.H. Kim, K.H. Jung, Y.K. Lee, K.H. Song, Metall. Mater. Trans. A Phys. Metall. Mater. 50(4), 2014 (2019)

[22] Z. F. Zhang, R. Liu, Z. J. Zhang, Y. Z. Tian, P. Zhang, Acta Metall. Sin. 54 (11), 1693-1704 (2018)

[23] S.A. Khairallah, A.T. Anderson, A. Rubenchik, W.E. King, Acta Mater. 108, 36 (2016)

[24] M.J. Matthews, G. Guss, S.A. Khairallah, A.M. Rubenchik, P.J. Depond, W.E. King, Acta Mater. 114, 33 (2016)

[25] C. Qiu, C. Panwisawas, M. Ward, H.C. Basoalto, J.W. Brooks, M.M. Attallah, Acta Mater. 96, 72 (2015)

[26] A. Pineau, D.L. McDowell, E.P. Busso, S.D. Antolovich, Acta Mater. 107, 484 (2016) 
[27] T. Zhai, A.J. Wilkinson, J.W. Martin, Acta Mater. 48(20), 4917 (2000)

[28] J.A. Hall, Int. J. Fatigue 19(1), S23 (1997)

[29] S. Das, Adv. Eng. Mater. 5(10), 701 (2003)

[30] A. Basak, S. Das, Annu. Rev. Mater. Res. 46(1), 125 (2016)

[31] L. Toth, S.Y. Yarema, Mater. Sci. 42(5), 673 (2006)

[32] H. Ohba, Y. Murakami, T. Endo, Trans. Jpn. Soc. Mech. Eng. 49(444), 901 (1983)
[33] Y. Murakami, J.S.M.E. Int, J. Ser. I 32(2), 167 (1989)

[34] J.C. Pang, S.X. Li, Z.G. Wang, Z.F. Zhang, Mater. Sci. Eng. A 564, 331 (2013)

[35] B. Wang, P. Zhang, R. Liu, Q.Q. Duan, Z.J. Zhang, X.W. Li, Z.F. Zhang, Mater. Sci. Eng. A 736, 105 (2018)

[36] M.X. Zhang, J.C. Pang, S.X. Li, Z.J. Zhang, Z.F. Zhang, Adv. Eng. Mater. 20(11), 1800554 (2018) 